\title{
Nonlinear Theory of Plasma-Beam Superheterodyne Free Electron Laser of Dopplertron Type with Non-Axial Injection of Electron Beam
}

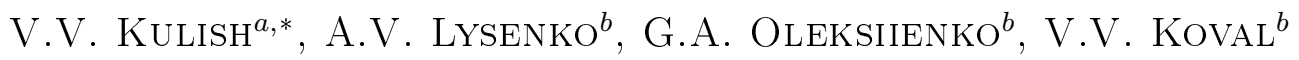 \\ AND M. YU. ROMBOVSKY ${ }^{b}$ \\ ${ }^{a}$ National Aviation University, Department of Theoretical Physics, Kiev, 03058, Ukraine \\ ${ }^{b}$ Sumy State University, Department of General and Theoretical Physics, 40007 Sumy, Ukraine
}

(Received July 11, 2014; in final form August 24, 2014)

\begin{abstract}
We have developed a quadratic nonlinear theory of a plasma-beam superheterodyne free electron laser of dopplertron type with the non-axial injection of an electron beam with respect to the guiding magnetic field. It is found out that the studied device can work in four different modes of operation. We determine the modes that have maximum gain coefficients of electromagnetic signals. It is shown that for all the operating modes the amplification factor of an electromagnetic signal is enhanced when the electron beam injection angle is increased.
\end{abstract}

DOI: 10.12693 /APhysPolA.126.1263

PACS: $41.60 . \mathrm{Cr}$

\section{Introduction}

Attention of researchers often attracts devices that generate and amplify a coherent electromagnetic radiation in the millimeter and submillimeter wavelength ranges [1-4]. Plasma-beam superheterodyne free electron lasers (SFEL) are the representatives of such systems $[1,5-8]$. The feature of the SFEL is the using of an additional mechanism to amplify one of the waves, which participate in a three-wave parametric resonance. Beam instabilities (e.g. double-stream, plasma-beam instabilities) are used as an additional amplification mechanism of the SFEL. Such instabilities are able to provide extremely high levels of beam wave amplification $[1,3,9]$. Therefore, SFELs have high amplifying properties and permanently capture the attention of scientists $[1,5-8,10-18]$.

First the idea of the plasma-beam SFEL (PBSFEL), which uses the plasma-beam instability as an additional amplification mechanism and the electromagnetic wave propagated in magnetized system as a pump, was proposed in [5]. Amplifying properties of the PBSFEL in the case of the axial electron beam injection with respect to the guiding magnetic field were studied in the framework of a cubic nonlinear theory in [6]. In paper [6] the mode in that the high frequency extraordinary (signal) $[1,3,9]$ wave and the low-frequency retarded (pump) electromagnetic wave participate in a three-wave parametric resonance was studied. Other modes that differ from previously studied ones, for example, using the ordinary electromagnetic wave [1, 3, 9] as a signal, using electromagnetic waves with other propagation directions have not been previously researched. Also it should be noted that dynamics of waves in the plasma-beam SFEL

*corresponding author; e-mail: kulish2001@ukr.net with non-axial injection of the electron beam with respect to the guiding magnetic field had not been previously considered. But, it is known from literature that in some cases the non-axial beam injection results in an increase in efficiency of other relativistic high power devices, for example two-stream SFELs [1-4, 11-13, 19].

The aims of this paper are research of different operation modes and study of the influence of the non-axial electron beam incidence on the amplifying properties of the PBSFEL.

\section{Model}

The model of the studied device is shown in Fig. 1. Plasma 1, which is characterized by the Langmuir frequency $\omega_{p}$, is located in the longitudinal focusing magnetic field with strength $H_{0}$. Relativistic electron beam 2 is injected in this plasma environment at an angle $\alpha$ with respect to the magnetic field strength vector. The Langmuir frequency of the electron beam is $\omega_{b}, \omega_{b} \ll \omega_{p}$. We chose a circularly polarized intense low-frequency electromagnetic wave with frequency $\omega_{2}$ and wave number $k_{2}$ as a pump. This wave propagates along the guiding magnetic field (axis $Z$ ) and in the opposite direction to the electron beam. The frequency of this wave is less than the cyclotron frequency $\omega_{H}$ of electron rotation in a magnetic field. We consider the case when $\omega_{H} \ll \omega_{p}$. Also we feed a weak high-frequency circularly polarized electromagnetic wave (signal wave) with frequency $\omega_{1}$ and wave number $k_{1}$ into the system.

The parametric resonance between the signal wave and the pump wave results in excitation of a space-charge wave $(\mathrm{SCW})$ with frequency $\omega_{3}$ and wave number $k_{3,1}$ of its first harmonic. The three-wave parametric resonance condition for the frequencies and wave numbers of first wave harmonics in this system has the form

$$
\omega_{3}=\omega_{1}-\omega_{2}, \quad k_{3}=k_{1}+k_{2} .
$$




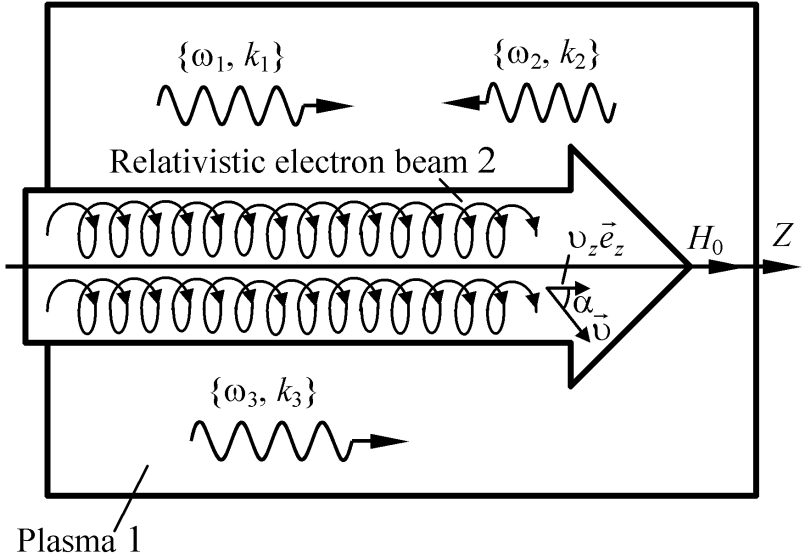

Fig. 1. Schematic diagram of the plasma-beam SFEL of dopplertron type.

It should be noted that the pump wave $\left(\omega_{2}, k_{2}\right)$ propagates in the opposite direction with respect to the axis $Z$ (Fig. 1) and therefore $k_{2}=-\left|k_{2}\right|$. The direction of the wave signal may be different, so the wave number $k_{1}$ can be positive or negative.

The superheterodyne amplification effect is used in the SFEL. Its essence is to use an additional mechanism to amplify one of the three waves, which are involved in the parametric resonance. In the studied device plasmabeam instability [1, 3, and 9] is used as an additional mechanism for SCW amplification. Growth increment of the $\mathrm{SCW}$ related to the plasma-beam instability is much greater than the growth increment related to the threewave parametric resonance. Therefore, the wave amplification in the PBSFEL is mainly due to the beam-plasma instability. The three-wave parametric resonance, in fact, is reduced only to transfer of the additional amplification from the space-charge wave on the electromagnetic wave signal $\left(\omega_{1}, k_{1}\right)$. Because of the fact that growth increments of the plasma-beam instability are high, the gain of the electromagnetic wave is also large enough. Therefore, we choose the system parameters so that the spacecharge wave in the studied system was growing due to plasma-beam instability, and the increment of growth of the SCW first harmonic was maximal.

Another feature of the PBSFEL is that the SCW, which is growing as a result of the plasma-beam instability, is characterized by a quasilinear dispersion relation. This means that frequencies and wave numbers $m$-th harmonics of the SCW are directly proportional to the harmonic number $m$ : $\omega_{3, m}=\omega_{3} m, k_{3, m} \approx k_{3,1} m$. This results in that a great number of three-wave resonance interactions between harmonics of the SCW appear in the plasma-beam system $[1,6,10]$. We take into account such resonant interactions in this paper.

\section{Basic equations}

As initial equations we use the relativistic quasihydrodynamic equation, the continuity equation and
Maxwell's equations. We use the hierarchical approach to a theory of oscillations and waves $[1,10]$. As a result of the standard procedure of hierarchical approach, we obtain the set of differential equations for the complex amplitude of $x$ - and $y$-component of the first harmonic signal $\left(E_{1 x, 1} ; E_{1 y, 1}\right), x$ - and $y$-component first harmonic pump $\left(E_{2 x, 1} ; E_{2 y, 1}\right)$ and the $m$-th harmonics $\left(E_{3 z, m}\right)$ SCW in the quadratic approximation

$$
\begin{aligned}
& K_{1} \frac{\mathrm{d} E_{1 x, 1}}{\mathrm{~d} t}+D_{1} E_{1 x, 1}=K_{3} E_{3 z, 1} E_{2 x, 1}, \\
& K_{1} \frac{\mathrm{d} E_{1 y, 1}}{\mathrm{~d} t}+D_{1} E_{1 y, 1}=K_{3} \eta_{1} \eta_{2} E_{3 z, 1} E_{2 y, 1}, \\
& M_{1} \frac{\mathrm{d} E_{2 x, 1}}{\mathrm{~d} t}+D_{2} E_{2 x, 1}=M_{3} E_{3 z, 1}^{*} E_{1 x, 1}, \\
& M_{1} \frac{\mathrm{d} E_{2 y, 1}}{\mathrm{~d} t}+D_{2} E_{2 y, 1}=M_{3} \eta_{1} \eta_{2} E_{3 z, 1}^{*} E_{1 y, 1}, \\
& C_{2, m} \frac{\mathrm{d}^{2} E_{3 z, m}}{\mathrm{~d} t^{2}}+C_{1, m} \frac{\mathrm{d} E_{3 z, m}}{\mathrm{~d} t}+D_{3, m} E_{3 z, m}= \\
& C_{3, m}\left(E_{1 x, m} E_{2 x, m}^{*}+E_{1 y, m} E_{2 y, m}^{*}\right)+F_{m}\left(E_{3 z}\right) .
\end{aligned}
$$

It follows from Eqs. (2)-(6) that the eigenwaves of this system are circularly polarized electromagnetic waves. In Eqs. (2)-(5) functions $D_{1}=D\left(\omega_{1}, k_{1}\right)$, $D_{2}=D\left(\omega_{2}, k_{2}\right)$ are the dispersion functions of the transverse electromagnetic circularly polarized signal $\left(\omega_{1}, k_{1}\right)$ and pump $\left(\omega_{2}, k_{2}\right)$ waves, where

$$
\begin{aligned}
& D_{j}\left(\omega_{j}, k_{j}\right)=\frac{\mathrm{i}}{c \omega_{j}}\left\{k_{j}^{2} c^{2}-\omega_{j}^{2}+\sum_{\alpha}^{b, p}\left[\frac{\omega_{\alpha}^{2}}{\gamma_{\alpha}\left(\Omega_{j, \alpha}+\eta_{j} \omega_{H, \alpha}\right)^{2}}\right.\right. \\
& \left.\left.\quad \times\left(\Omega_{j, \alpha}\left(\Omega_{j, \alpha}+\eta_{j} \omega_{H, \alpha}\right)-\frac{v_{\alpha \perp}^{2}}{2 c^{2}}\left(\omega_{j}^{2}-k_{j}^{2} c^{2}\right)\right)\right]\right\}, \\
& \quad(j=1,2) . \\
& \eta_{1}=E_{1 y, 1} /\left(\mathrm{i} E_{1 x, 1}\right)= \pm 1, \eta_{2}=E_{2 y, 1} /\left(\mathrm{i} E_{2 x, 1}\right)= \pm 1
\end{aligned}
$$

are the sign functions characterizing the rotation direction of the electric field strength of the circularly polarized electromagnetic waves; $c$ is the speed of light; $\gamma_{\alpha}=1 / \sqrt{1-\left(v_{\alpha z}^{2}+v_{\alpha \perp}^{2}\right) / c^{2}}$ is the relativistic factor (the subscript $\alpha$ accepts the values $b$ and $p ; b$ is the index characterizing the beam parameters, $p$ is the plasma parameter index); $v_{\alpha z}$ and $v_{\alpha \perp}$ are, respectively, the longitudinal and the transversal velocity of the electrons; $\Omega_{j, \alpha}=\omega_{j}-k_{j} v_{\alpha z} ; \omega_{H, \alpha}=e H_{0} /\left(m_{e} c \gamma_{\alpha}\right)$ is the cyclotron frequency of electron rotation in a longitudinal magnetic field; $e$ is the electron charge; $m_{e}$ is the electron mass; $K_{1}=\partial D_{1} / \partial\left(i \omega_{1}\right) ; M_{1}=\partial D_{2} / \partial\left(i \omega_{2}\right)$. In Eq. (6)

$$
D_{3, m}=\frac{-\mathrm{i} m \omega_{3}}{c}\left(1-\sum_{\alpha}^{b, p} \frac{\omega_{\alpha}^{2}\left(1-v_{\alpha z}^{2} / c^{2}\right)}{\left(m \omega_{3}-k_{3, m} v_{\alpha z}\right)^{2} \gamma_{\alpha}}\right)
$$

is the dispersion function of $\mathrm{SCW} ; C_{1, m}=$ $\partial D_{3, m} / \partial\left(i m \omega_{3}\right) ; \quad C_{2, m}=0.5 \partial^{2} D_{3, m} / \partial\left(\mathrm{i} m \omega_{3}\right)^{2}$. $K_{3}, M_{3}, C_{3, m}$ are the differential equation coefficients depending on frequencies, wave numbers and parameters of the studied system; $F_{m}\left(E_{3 z}\right)$ is the function, which is determined by the plural parametric resonance interactions between the harmonics SCW and depends on these harmonics $[1,6,10]$. 
As it has been mentioned above, one of interacting waves $(\mathrm{SCW})$ is amplified by the plasma-beam instability. To consider this additional amplification, we take into account the term $C_{2, m} d^{2} E_{3 z, m} / \mathrm{d} t^{2}$ in Eq. (6). Through this in the case of absence of the parametric resonances between the SCW, the signal and pump waves the SCW increment of growth will be determined by a plasmabeam instability and will be approximately determined by the expression $\left(-D_{3, m} / C_{2, m}\right)^{1 / 2}$ (here we suppose that $\left.C_{2, m} \mathrm{~d}^{2} E_{3 z, m} / \mathrm{d} t^{2}, D_{3 m} E_{3 z, m} \gg C_{1, m} \mathrm{~d} E_{3 z, m} / \mathrm{d} t\right)$. It should be noted that in this case the dispersion equation for the SCW has complex roots. So when we substitute real frequencies and real wave numbers in the dispersion function $D_{3, m}\left(\omega_{3}, k_{3}\right)$ we obtain $D_{3, m} \neq 0$.

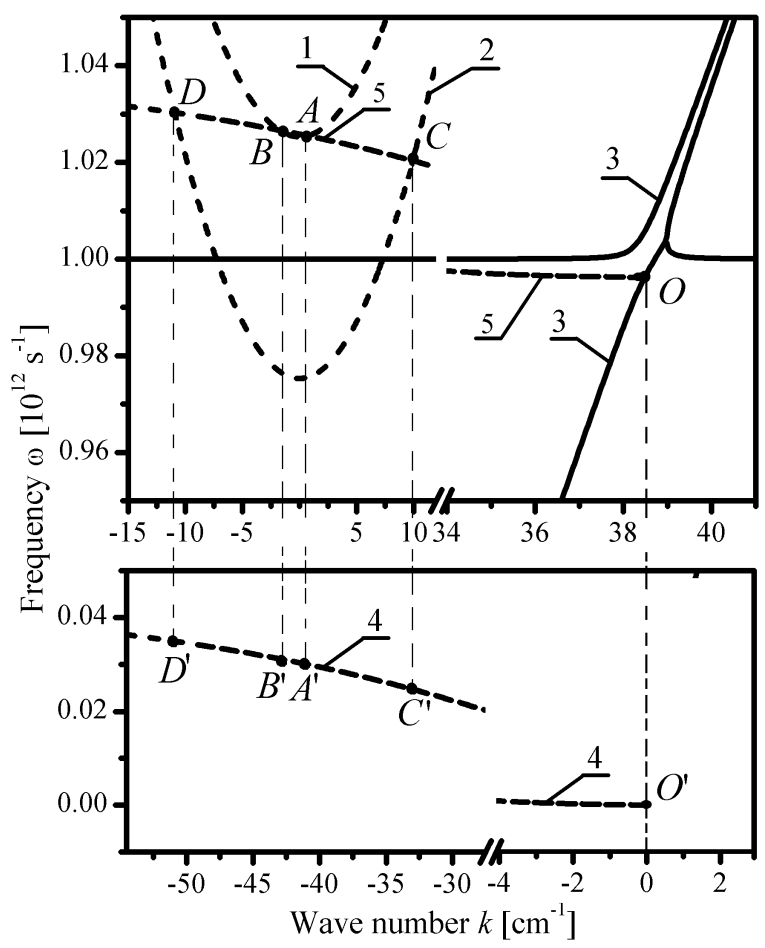

Fig. 2. Dispersion curves of the signal high-frequency electromagnetic waves (curves 1,2) and the space charge wave (curves 3 ) in the plasma-beam SFEL, which were calculated for a system with the parameters given in Table.

As it has already mentioned above, the dispersion relation for the wave SCW is quasilinear (Fig. 2, curve 3). This results in the fact that $\mathrm{SCW}$ harmonics interact with one another through the mechanism of the threewave parametric resonance interactions. Let us denote numbers of interacting harmonics by $m_{1}, m_{2}$, and $m_{3}$. Then the condition of the three-wave parametric resonance for these harmonics takes the form

$$
\omega_{3, m 1}=\omega_{3, m 2}+\omega_{3, m 3}, \quad k_{3, m 1}=k_{3, m 2}+k_{3, m 3} .
$$

We take into account that the dispersion relation is quasilinear $\left(\omega_{3, m}=\omega_{3} m, k_{3, m} \approx k_{3,1} m\right)$. Then condition $(10)$ transformed into

$$
m_{1}=m_{2}+m_{3} \text {. }
$$

It should be recalled that $m_{1}, m_{2}$, and $m_{3}$ are integers. We can fulfil the condition (11) in a variety of ways, for example $2=1+1,3=1+2$ and so on. Such threewave interactions result in generation and amplification of the higher harmonics of the SCW. Function $F_{m}\left(E_{3 z}\right)$ in Eq. (6) describes this effect. We note that the effect of this term in the quadratic approximation is slight, whereas the cubic effect of this term could be significant.

Also, the set of Eqs. (2)-(6) describes the parametric instability of the first harmonics of signal, pump and $\mathrm{SCW}$ waves. If in the considered device the plasma-beam instability was absent, and plural interactions between the harmonics wave SCW could be neglected, then the wave signal growth would be only provided by the threewave parametric interaction of signal, pump and SCW. In this case we can easily determine the growth increment of the wave from the set of Eqs. (2)-(6) accepted that $C_{2, m} \mathrm{~d}^{2} E_{3 z, m} / \mathrm{d} t^{2}=0, D_{3 m} E_{3 z, m}=0, F_{m}\left(E_{3 z}\right)=0$ and amplitude of the pump wave is constant. Assuming $E_{1 x, 1}, E_{1 y, 1}, E_{3 z, 1} \sim \exp \left(\alpha_{\mathrm{par}} t\right)$ we find that

$$
\alpha_{\text {par }}=\sqrt{\frac{K_{3} C_{3,1}\left(1+\eta_{1} \eta_{2}\right)}{K_{1} C_{1,1}}\left|E_{2 x, 1}\right|^{2}} .
$$

This result indicates that if the circularly polarized electromagnetic pump and signal waves have different rotation directions of the electric field strength vectors, i.e. $\eta_{1} \eta_{2}=-1$, the parametric increment of growth will be equal to zero $\alpha_{\mathrm{par}} \sim\left(1+\eta_{1} \eta_{2}\right)^{0.5}=0$ and the parametric instability will be absent. Thus, for implementation of the parametric resonance it is necessary that the electromagnetic signal and the pump waves have the same rotation directions of the electric field strength vector $\eta_{1} \eta_{2}=+1$. This means that rotation of the electric field strength vectors of both waves should be clockwise $\left(\eta_{1}=-1, \eta_{2}=-1\right)$ or counterclockwise $\left(\eta_{1}=+1\right.$, $\left.\eta_{2}=+1\right)$ viewing along the magnetic field strength vector. This conclusion completely agrees with the conclusions of the general theory in relation to resonant polarization [1].

It should be noted that the resulting wave amplification in PBSFEL is defined as the gain due to the plasma-beam instability, and the amplification due to the three-wave parametric resonance. The plasma-beam instability contribution to the resulting wave amplification is determinant.

\section{Analysis}

Let us analyze dynamics of waves in the PBSFEL with parameters which are presented in Table. Let us find frequencies and wave numbers of the signal, pump and SCW waves, which participate in the three-wave parametric resonance in the studied device. For this purpose, we draw the dispersion curves for the first harmonics of these waves in Fig. 2, using dispersion relations $D_{1}\left(\omega_{1}, k_{1}\right)=0$, $D_{2}\left(\omega_{2}, k_{2}\right)=0$ and $D_{3,1}\left(\omega_{3}, k_{3,1}\right)=0$, where the functions $D_{j}\left(\omega_{j}, k_{j}\right)$ are defined by $(7)$, and the function $D_{3,1}\left(\omega_{3}, k_{3,1}\right)$ is defined by (9). Figure 2 shows the dispersion curves for the high-frequency electromagnetic 
signal waves $\left(\left(\omega_{1}, k_{1}\right)\right.$, curves 1 and 2$)$, low-frequency electromagnetic pump waves $\left(\left(\omega_{2}, k_{2}\right)\right.$, curves 4$)$ and the $\mathrm{SCW}\left(\left(\omega_{3}, k_{3,1}\right)\right.$, curves 3$)$ for the case when the injection angle of the beam with respect to the longitudinal magnetic field strength is equal to zero.

TABLE

Parameters of the studied plasma-beam superheterodyne free electron laser.

\begin{tabular}{l|l}
\hline \hline Parameters & Magnitudes \\
\hline Langmuir frequency of the plasma $\omega_{p}\left[\mathrm{~s}^{-1}\right]$ & $1.0 \times 10^{12}$ \\
Langmuir frequency of the beam $\omega_{b}\left[\mathrm{~s}^{-1}\right]$ & $2.0 \times 10^{9}$ \\
Energy of the beam [MeV] & 0.51 \\
Focusing magnetic field [G] & $2.8 \times 10^{3}$ \\
The amplitude of the first harmonic & $2.8 \times 10^{4}$ \\
of the pump electric field is & \\
$E_{2,1}=\sqrt{\left|E_{2 x, 1}\right|^{2}+\left|E_{2 y, 1}\right|^{2}}[\mathrm{~V} / \mathrm{m}]$ &
\end{tabular}

Curve 1 corresponds to a right-hand circularly polarized electromagnetic signal wave viewing along the magnetic field strength vector $\left(\eta_{1}=-1\right)$, curve 2 corresponds to a left-hand circularly polarized wave $\left(\eta_{1}=+1\right)$.

Curve 4 corresponds to both a right-hand circularly polarized electromagnetic pump wave $\left(\eta_{2}=-1\right)$ and a left-hand circularly polarized pump wave $\left(\eta_{2}=+1\right)$. We choose wave number $k_{3,1}$ and frequency $\omega_{3}$ of the first SCW harmonic so that this harmonic was characterized by maximum increment of growth due to the plasma-beam instability. As it is known, wave number value $k_{3,1}=\omega_{p} / v_{b z}$ corresponds to this condition [1-3]. Frequency $\omega_{3}$ is determined from equation $D_{3,1}\left(\omega_{3}, k_{3,1}\right)=0$. These values of the wave number $k_{3,1}$ and the frequency $\omega_{3}$ correspond to point $O$ on the dispersion curve SCW 3.

To determine the electromagnetic wave frequencies $\omega_{1}, \omega_{2}$ of the signal and the pump, as well as their wave numbers $k_{1}$ and $k_{2}$, it is necessary to solve the set of equations, which consists of three-wave parametric resonance conditions (1) (two equations), equation $D_{1}\left(\omega_{1}, k_{1}\right)=0$ (we use the solution that corresponds to the high-frequency electromagnetic wave), equation $D_{2}\left(\omega_{2}, k_{2}\right)=0$ (we use the solution that corresponds to the low-frequency electromagnetic wave). These four equations can be used to determine $\omega_{1}, k_{1}, \omega_{2}$, and $k_{2}$. These solutions are easy to find graphically, if in Fig. 2 we perform translation of curve 4 into curve 5 so that point $O^{\prime}$ has moved to point $O$. Then the intersections curve 5 with the curves 1 and 2 give the required values of the frequencies $\omega_{1}$ and the wave numbers $k_{1}$ of the high-frequency wave signal electromagnetic wave.

The points $A, B, C$ and $D$ define the required frequencies and the wave numbers. To determine the frequencies and the wave numbers of the low-frequency electromagnetic pump wave it is necessary to project points $A, B$, $C$ and $D$ onto the curve 4 in the translation direction. The points $A^{\prime}, B^{\prime}, C^{\prime}$ and $D^{\prime}$ define the frequencies $\omega_{2}$ and the wave numbers $k_{2}$. As can be seen from Fig. 2, the electromagnetic signal waves are propagated along the axis $Z$ in cases $A$ and $C$. In cases $B$ and $D$ they are propagated in the opposite direction. Thus, the threewave parametric interactions in the studied SFEL are possible in four different cases. It should be pointed out that previously only one operation mode of plasma-beam SFEL of dopplertron type has been studied. The extraordinary wave (curve 1 , point $A$ ) participates in this mode of operation $[7,8$, etc.]. Modes of operation $B, C$, and $D$ previously were not studied.

Using standard numerical methods, we analyze the dynamics of waves in the researched device for various operating modes of PBSFEL by means of Eqs. (2)-(6). Figure 3 shows the dependencies of the amplitudes of the first harmonic of the signal wave electric field on the normalized time $\tau=t \delta \omega$ ( $\delta \omega$ is the growth rate of the plasma-beam instability $[1,3$, and 9]) for the four possible modes of operation. Curve $A$ corresponds to mode $A$ (Fig. 2), curve $B$ corresponds to mode $B$, curve $C$ corresponds to mode $C$, curve $D$ corresponds to mode $D$. It is seen that the gain factors of the signal waves are approximately identical for modes $A$ and $B$, which correspond to the interaction with an extraordinary signal wave $\left(\eta_{1}=-1\right)$. The gain factors of the signal wave are also approximately identical for modes $C$ and $D$, which correspond to the interaction with the ordinary signal wave $\left(\eta_{1}=+1\right)$. However, curves $C$ and $D$ essentially differ from curves $A$ and $B$.

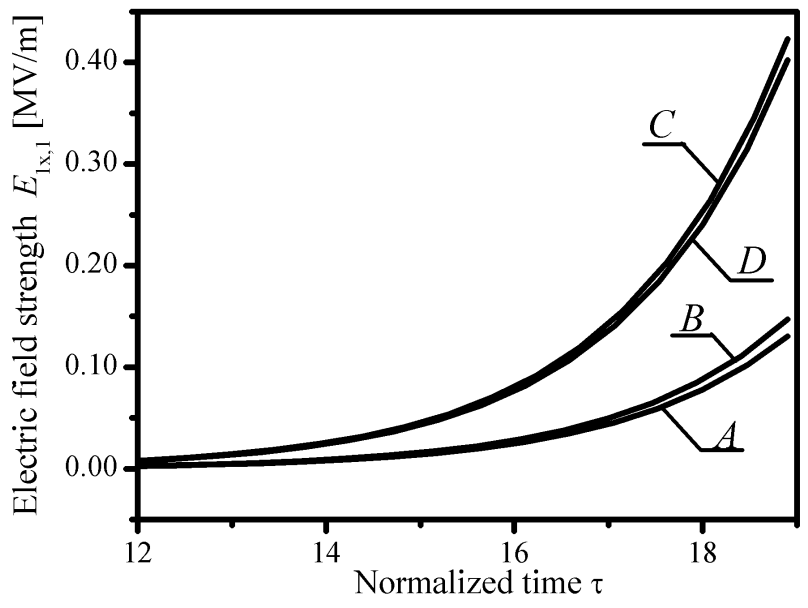

Fig. 3. Amplitudes of the first harmonic of the signal wave electric field as a function of normalized time $\tau=t \delta \omega$. Curve $A$ corresponds to mode $A$, curve $B$ corresponds to mode $B$, curve $C$ corresponds to mode $C$, curve $D$ corresponds to mode $D$ (Fig. 2).

From Fig. 3 it follows that mode $C$ has a maximum gain of the signal wave. In this case, it is necessary to consider that the increment of growth of the SCW (the frequency and the wave number of the SCW are characterized by the point $O$ in Fig. 2) due to the plasma-beam instability has the same value in all four modes. But the parametric increments of growth $\alpha_{\text {par }}$ (12) for modes $A$, $B, C$, and $D$ are different. It is the parametric increment 
of growth that determines different dynamics of the signal wave in the different modes of operation, which is shown in Fig. 3.

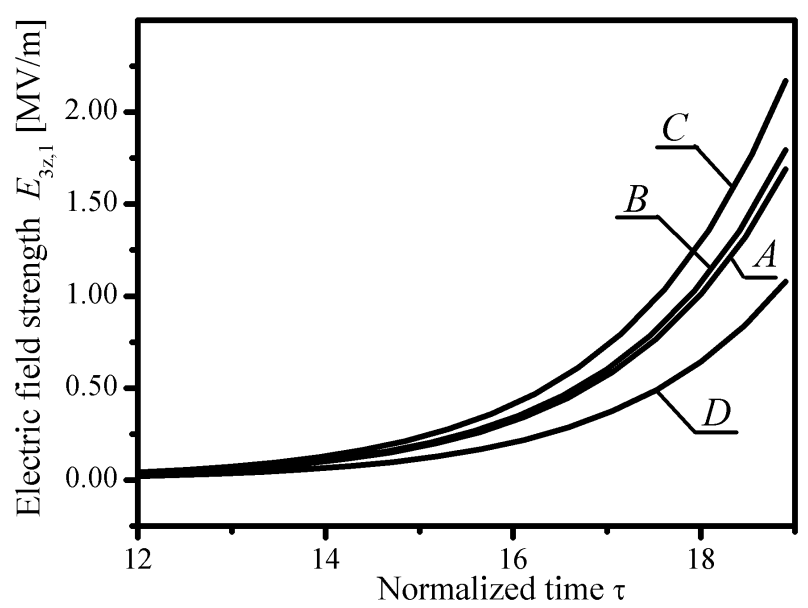

Fig. 4. Amplitudes of the first harmonic of the SCW electric field as a function of normalized time $\tau=t \delta \omega$. Curve $A$ corresponds to the interaction mode $A$, curve $B$ to mode $B$, curve $C$ to mode $C$, curve $D$ to mode $D$ (Fig. 2).

Figure 4 shows the dependences of the amplitudes of the first harmonic of the SCW electric field as a function of normalized time $\tau=t \delta \omega$. We see that the maximum of the gain SCW takes place, as in the case of the signal wave (Fig. 3 ), for mode $C$. The SCW amplification in mode $D$ is minimal, whereas in modes $A$ and $B$ it is practically the same. Such behavior of the curves is explained by the different values of the coefficient $C_{3,1}$ in Eq. (8) for the different modes of wave's interaction.

Figure 5 shows the dependences of the amplitudes of the first harmonic of the signal wave electric field on the normalized time $\tau=t \delta \omega$ at different injection angles of the beam $\alpha$ with respect to the magnetic field for mode of interaction $C$. Curve 1 corresponds to the injection angle $\alpha=0^{\circ}$, curve 2 corresponds to $\alpha=10^{\circ}$, curve 3 corresponds to $\alpha=20^{\circ}$, curve 4 corresponds to $\alpha=30^{\circ}$. It follows from Fig. 5 that the gain coefficient of the signal wave grows with increasing of the angle $\alpha$. First of all this effect is associated with the change of the increment of growth of the plasma-beam instability. At the same time, the parametric increment of growth changes inessentially. The increment of growth of plasma-beam instability can be found from the equation $D_{3, m}=0$, where $D_{3, m}$ is defined by Eq. (9). From this equation follows that $D_{3, m}$ depends on the longitudinal beam velocity $v_{b z}$. In the case of the non-axial beam injection the longitudinal beam velocity decreases (it should be noted that the beam energy remains constant, $\gamma_{b}=$ const). This results in a growth of the increment of growth of plasma-beam instability and, as a consequence, in an increase of the gain of the electromagnetic signal wave. This effect occurs in all four operation modes of the plasma-beam SFEL.

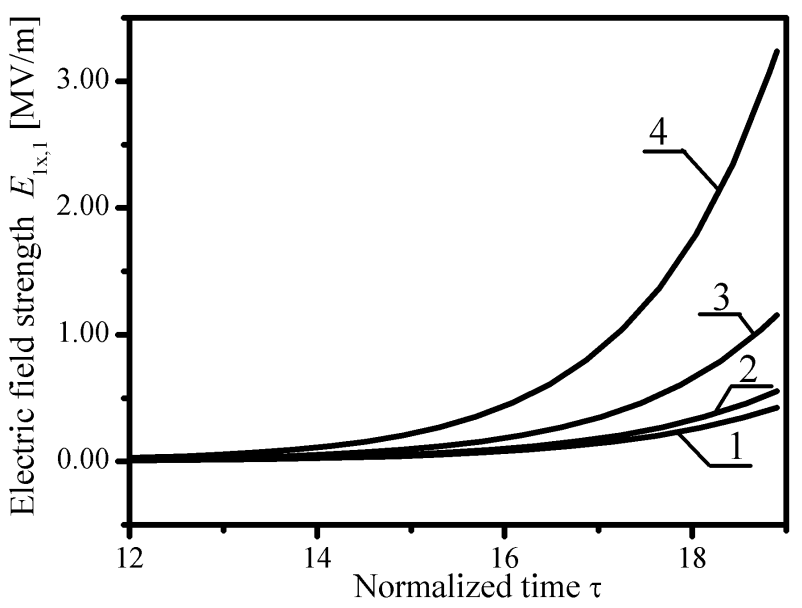

Fig. 5. Amplitudes of the first harmonic of the signal wave electric field as a function of normalized time $\tau=t \delta \omega$ at different injection angles of the electron beam $\alpha$ with respect to the magnetic field. Curve 1 corresponds to the injection angle $\alpha=0^{\circ}$, curve 2 corresponds to $\alpha=10^{\circ}$, curve 3 corresponds to $\alpha=20^{\circ}$, curve 4 corresponds to $\alpha=30^{\circ}$. The curves correspond to operating mode $C$.

\section{Conclusions}

We have constructed the quadratic nonlinear theory of the plasma-beam superheterodyne free electron laser of dopplertron type with the non-axial injection of an electron beam with respect to the guiding magnetic field. It is found that four different modes of operation can be realized in the researched device. Ordinary transverse electromagnetic waves take part in a three-wave parametric resonance in two modes. Extraordinary transverse electromagnetic waves participate in other two modes. It is demonstrated that the modes of operation involved with the ordinary electromagnetic waves are characterized by maximum gains of signal waves. We have analyzed the influence of the injection angle of an electron beam on the dynamics of waves in the SFEL. It is shown that the electromagnetic signal gain increases when the beam injection angle is enhanced for all the modes of operation. It is found out that such the increase of the signal gain is determined by the rise of a plasma-beam instability increment of growth.

\section{References}

[1] V.V. Kulish, Hierarchic Electrodynamics and Free Electron Lasers, CRC Press, Boca Raton 2011.

[2] S.E. Tsimring, Electron Beams and Microwave Vacuum Electronics, Wiley, Hoboken (NJ) 2007.

[3] M.V. Kuzelev, A.A. Ruhadze, P.S. Strelkov, The Relativistic Plasma SHF Electronic, Bauman MSTU, Moscow 2002.

[4] J.H. Booske, R.J. Dobbs, C.D. Joye, C.L. Kory, G.R. Neil, Gun-Sik Park, Park Jaehun, R.J. Temkin, IEEE Trans. Terahertz Sci. Technol. 1, 54 (2011).

[5] N. Ya. Kotsarenko, V. V. Kulish, Radiotekh. Elektron 25, 2470 (1980). 
[6] V.V. Kulish, A.V. Lysenko, V.V. Koval, Plasma Phys. Rep. 36, 1185 (2010).

[7] V.V. Kulish, A.V. Lysenko, V.V. Koval, Telecommun. Radio Eng. 69, 1859 (2010).

[8] V.V. Kulish, A.V. Lysenko, V.V. Koval, Techn. Phys. Lett. 35, 696 (2009).

[9] N.A. Krall, A.W. Trivelpiece, Principles of Plasma Physics, San Francisco Press, New York 1986.

[10] V.V. Kulish, A.V. Lysenko, A.Ju. Brusnik, J. Infrared Millim. Terahertz Waves 33, 149 (2012).

[11] T. Mohsenpour, N. Mehrabi, Phys. Plasmas 20, 082133 (2013).

[12] S. Saviz, Z. Rezaei, F.M. Aghamir, Chin. Phys. B 21, 094103 (2012).

[13] W. Liu, Z. Yang, Z. Liang, Int. J. Infrared Millim. Waves 27, 1073 (2006).

[14] N. Mahdizadeh, F.M. Aghamir, J. Appl. Phys. 113 , 083305 (2013).
[15] S. Saviz, M. Karimi, Chin. Phys. B 23, 034103 (2014).

[16] B.W.J. McNeil, G.R.M. Robb, Phys. Rev. E 70 , 035501 (2004).

[17] H. Mehdian, N. Abbasi, Phys. Plasmas 15, 013111 (2008).

[18] D.V. Rose, T.C. Genoni, D.R. Welch, E.A. Startsev, R.C. Davidson, Phys. Rev. ST Accel. Beams 10, 034203 (2007).

[19] M.K.A. Thumm, A.V. Arzhannikov, V.T. Astrelin, A.V. Burdakov, I.A. Ivanov, P.V. Kalinin, I.V. Kandaurov, V.V. Kurkuchekov, S.A. Kuznetsov, M.A. Makarov, K.I. Mekler, S.V. Polosatkin, S.A. Popov, V.V. Postupaev, A.F. Rovenskikh, S.L. Sinitsky, V.F. Sklyarov, V.D. Stepanov, Yu.A. Trunev, I.V. Timofeev, L.N. Vyacheslavov, J. Infrared Millim. Terahertz Waves 35, 81 (2014). 